Deformable Nanolaminate Optics

Scot S. Olivier, Alexandros P. Papavasiliou, Troy W. Barbee, Robin R. Miles, Chris C. Walton, Michael B. Cohn, Kevin Chang

May 15, 2006

Astronomical Telescopes and Instrumentation Orlando, FL, United States

May 24, 2006 through May 31, 2006 
This document was prepared as an account of work sponsored by an agency of the United States Government. Neither the United States Government nor the University of California nor any of their employees, makes any warranty, express or implied, or assumes any legal liability or responsibility for the accuracy, completeness, or usefulness of any information, apparatus, product, or process disclosed, or represents that its use would not infringe privately owned rights. Reference herein to any specific commercial product, process, or service by trade name, trademark, manufacturer, or otherwise, does not necessarily constitute or imply its endorsement, recommendation, or favoring by the United States Government or the University of California. The views and opinions of authors expressed herein do not necessarily state or reflect those of the United States Government or the University of California, and shall not be used for advertising or product endorsement purposes. 


\title{
Deformable Nanolaminate Optics
}

\author{
Scot S. Olivier ${ }^{\mathrm{a}}$, Alexandros P. Papavasiliou, Troy W. Barbee ${ }^{\mathrm{a}}$, Robin R. Miles ${ }^{\mathrm{a}}$, Chris C. Walton ${ }^{\mathrm{a}}$, \\ Michael B. Cohn ${ }^{\mathrm{b}}$, Kevin Chang ${ }^{\mathrm{b}}$ \\ ${ }^{a}$ Lawrence Livermore National Laboratory, 7000 East Ave. Livermore CA 94550 \\ ${ }^{\mathrm{b}}$ MicroAssembly Technologies, 3065 Richmond Parkway Suite 109, Richmond CA 94806
}

\begin{abstract}
We are developing a new class of deformable optic based on electrostatic actuation of nanolaminate foils. These foils are engineered at the atomic level to provide optimal opto-mechanical properties, including surface quality, strength and stiffness, for a wide range of deformable optics. We are combining these foils, developed at Lawrence Livermore National Laboratory (LLNL), with commercial metal processing techniques to produce prototype deformable optics with aperture sizes up to $10 \mathrm{~cm}$ and actuator spacing from $1 \mathrm{~mm}$ to $1 \mathrm{~cm}$ and with a range of surface deformation designed to be as much as 10 microns. The existing capability for producing nanolaminate foils at LLNL, coupled with the commercial metal processing techniques being used, enable the potential production of these deformable optics with aperture sizes of over $1 \mathrm{~m}$, and much larger deformable optics could potentially be produced by tiling multiple deformable segments. In addition, based on the fabrication processes being used, deformable nanolaminate optics could potentially be produced with areal densities of less than $1 \mathrm{~kg}$ per square $\mathrm{m}$ for applications in which lightweight deformable optics are desirable, and deformable nanolaminate optics could potentially be fabricated with intrinsically curved surfaces, including aspheric shapes. We will describe the basic principles of these devices, and we will present details of the design, fabrication and characterization of the prototype deformable nanolaminate optics that have been developed to date. We will also discuss the possibilities for future work on scaling these devices to larger sizes and developing both devices with lower areal densities and devices with curved surfaces.

Keywords: adaptive optics, active optics, deformable mirrors, lightweight optics, metal optics
\end{abstract}

\section{INTRODUCTION}

Deformable mirror are needed for use in optical systems ranging from large space optics, on the order of a meter or more, to small-scale mirrors for instruments on the order of a few millimeters. We are developing a mirror technology using nanolaminate foils that can be used to make mirrors with thousands of actuators over this wide range of scales.

\subsection{Previous MEMS Deformable mirrors}

Microfabrication has allowed the production of small deformable mirrors with very dense actuation. While these devices provide sufficient stroke, and high actuator density, they none of them are capable of being scaled beyond the size of a silicon wafer ${ }^{1,2}$ and many of them are limited to a single chip ${ }^{3}$.

\subsection{Nanolaminates}

Nanolaminates are thin, flexible, optically smooth metal foils with very high strength. Nanolaminate mirrors have been developed to as large, light-weight, passive optical elements ${ }^{4}$. However, their mechanical properties make them a good match for the high density but small force provided by electrostatic actuators. The high strength of nanolaminates makes it possible to produce a foil with a high quality optical surface that is simultaneously robust enough to survive fabrication and thin enough to be readily deformed by weak electrostatic actuators.

A nanolaminate foil is a metal foil created by sputtering thin layers of metal one on top of the other. The foils are deposited on a mandrell with a release layer that allows them to be parted from the mandrel. The with a sufficiently thin and conformal release layer, the foils take on the shape of the mandrel, and an optically smooth mandrel yields an optically smooth foil. The layers are deposited by magnetron sputtering over a moving table. The thickness of each individual layer as well as the whole thickness of the foil can be accurately controlled. The individual layers can be controlled to have thicknesses on the order of $1-10 \mathrm{~nm}$. The very thin layers restrict the growth of grains. The nanoscale grain size enforced by these thin layers produces a material dense with grain-boundry dislocations resulting in a very high strength. Since the material has very high strength, thin sheets of nanolaminate can be handled and will survive 
microfabrication. These foils can be made thin enough to allow them to be deformed by very weak electrostatic actuators but still strong enough to be handled and bonded to those actuators.

\section{DEVICE ARCHITECTURES}

The devices consist of three horizontal layers separated by vertical standoffs. The base is a stiff layer that provides mechanical support to the whole device. The spring layer is suspended above the base layer on top of ridges. The mirror layer is attached to the spring layer with posts. When a voltage is applied between the spring layer and electrodes on the base layer, the spring layer deforms to close the gap. This deformation is translated through the posts to the mirror layer. In the prototypes described here, the base layer is made of silicon or glass, but it could be fabricated from any stiff material such as a thick nanolaminate foil. The ridges and posts described here are fabricated from SU-8 epoxy or electroplated nickel. The mirror and spring layers are fabricated of either nanolaminate foil or rolled steel and invar foils.

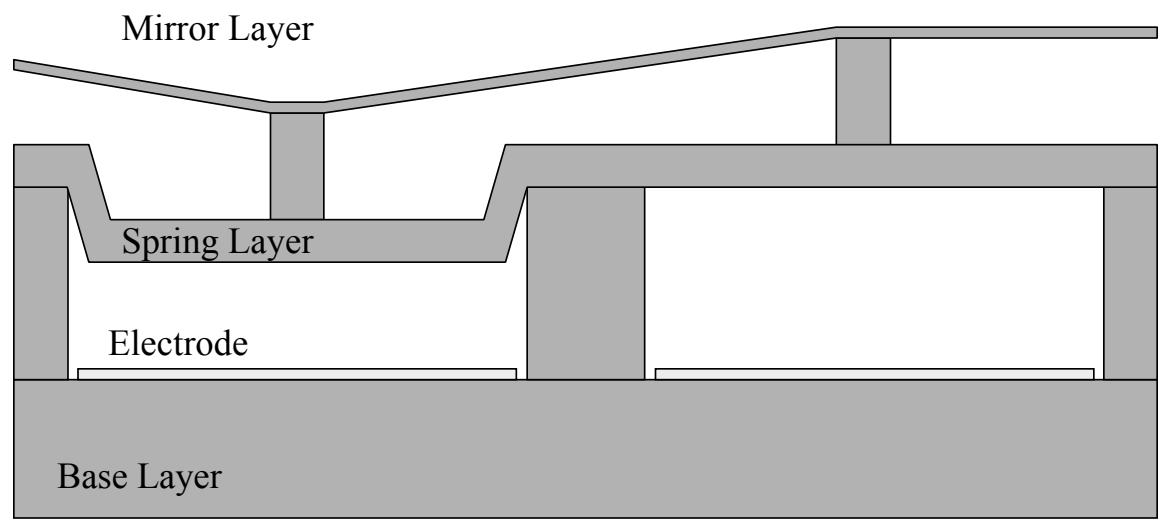

Figure 1. Cross-section of basic design.

\subsection{Actuator designs}

Two different types of spring layers have been used in this work: patterned springs, and a continuous spring layer. In the case of the patterned springs, beam shaped springs are cut into a fairly thick foil such that most of the spring layer moves in one rigid piece. In the other case, the spring layer is a thin, continuous piece of foil which deforms continuously.

The continuous design has the advantage that it does not require an additional patterning and aligning step. In addition continuous design is stable in a larger fraction of the initial gap than the patterned design. This increased stability comes as a result of the non-linearity of a deforming plate, and the fact that the portion of the plate near the edges does not move as far as the portion in the center, resulting in less overall force that the patterned actuator in which the actuator moves as a rigid plate. This disparity in overall force, however ultimately means that the patterned design requires less voltage for the same performance. 


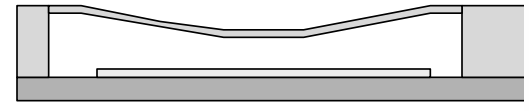

Continuous spring layer

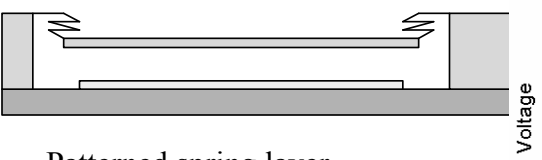

Patterned spring layer

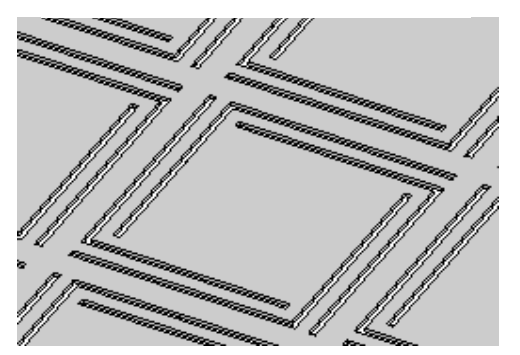

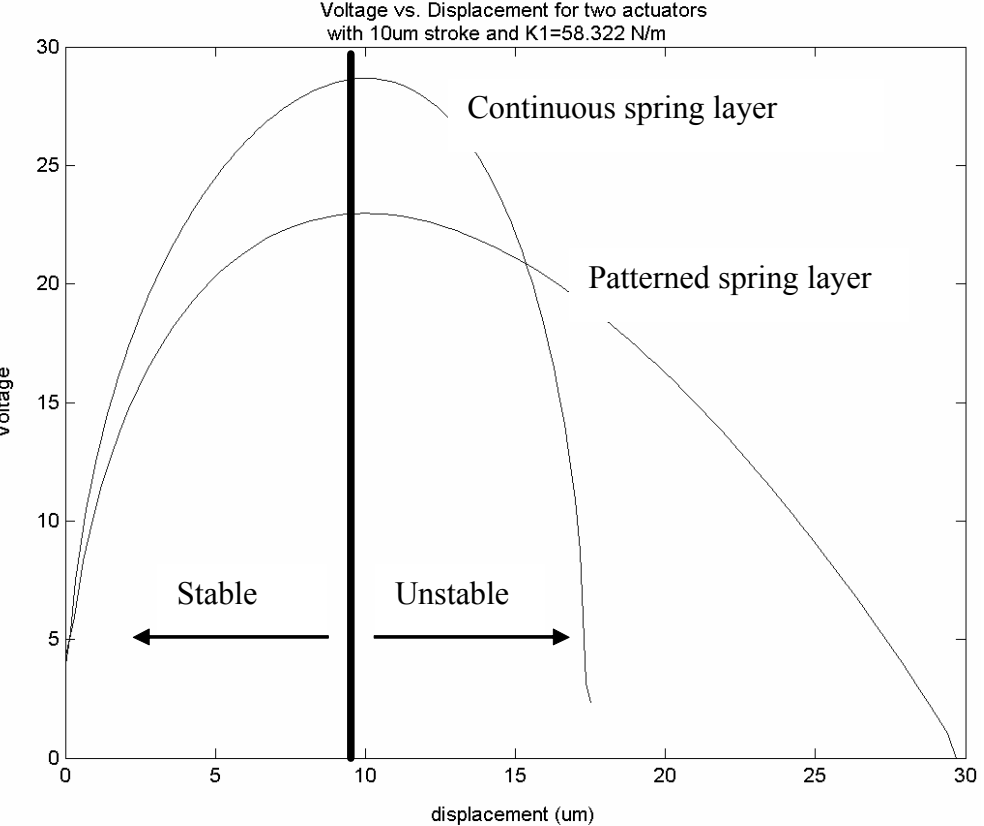

Figure 2. On the left are cross sections of the continuous and patterned spring layers as well as a drawing of the patterned spring layer design. On the right the voltage vs. displacement curve for parallel-plate with linear spring (patterned spring layer) and built-in plate (continuous spring layer) actuators. The actuators both have the same stroke $(10 \mu \mathrm{m})$ and the same spring constant. The actuators are only stable below $10 \mu \mathrm{m}$ displacement where an increase in voltage is required for an increase in displacement. The continuous spring layer only requires a $17 \mu \mathrm{m}$ gap, but requires more voltage than the patterned spring layer which requires a $30 \mu \mathrm{m}$ gap.

\subsection{Scalability}

An important goal of this project is to produce a technology that can be fabricated over a wide range of size scales. The smaller devices can be fabricated with the same microfabrication technologies used to fabricate conventional MEMS structures. However, larger devices require fabrication techniques that scale to those larger sizes. To accomplish MEMS-like functionality over a wide range of sizes we have used a combination of scaleable techniques and scaleappropriate techniques.

\subsubsection{Lithography}

We have used scale-appropriate techniques to pattern the deformable mirrors. Small scale mirrors have been patterned with conventional contact microlithography. Since the prototype large scale devices are also no larger than a silicon wafer, we have also used conventional microlithography, but designed the process to be compatible with printed circuit board lithography. Since the pitch of the actuators in these devices is proportionately larger, these devices are much more tolerant of lithography errors. We simulated the deviation in drive voltage as a function of deviations from the nominal actuator area and found that a lithography error of $50 \mu \mathrm{m}$, larger than those expected with dry-film resist lithography, produced a drive voltage deviation of only $0.8 \%$

\subsubsection{Electroplating}

Like the lithography of silicon MEMS devices, the production of consistent silicon films and the patterning of those films is limited to the size of silicon wafers. In order to fabricate devices on a much larger scale, we used electroplated metal to produce the vertical elements of the DM. The metal is electroplated up through a photoresist mask, then planarized to control the thickness of the posts and ridges. The thickness of the devices produced to date have variations on the order of $2-3.5 \mu \mathrm{m}$. This should result in $10 \%-15 \%$ drive voltage variation.

To date we have produced metal features for devices ranging from $2 \mathrm{~cm}$ wide to a device that nearly fills a 4 " wafer. While to-date only 4" wafer sized samples have been produced, the technology exists to extend this production technique to larger scales. Very precise thickness control can be achieved by a combination of localized addition and 
removal of material along with localized thickness measurements. Brush plating can be used to add material locally by concentrating the field and plating solution in a localized area where not enough material had been deposited earlier. Small-tool grinding can be used to remove material from areas where excess material has been deposited.

\subsubsection{Metal Foils for springs and mirrors}

The horizontal spring and mirror layers in these devices have been fabricated from rolled metal foils or nanolaminate foils. Both of these materials can be fabricated over a wide range of sizes. Rolled metal foils are commercially available in very large sizes. The nanolaminates used in this project are deposited on 4 and 6 inch diameter substrates. However, there is no fundamental limit on the size of a nanolaminate foil. Currently nanolaminate foils up to $75 \mathrm{~cm}$ in diameter are being developed at LLNL in conjunction with JPL and the NASA ${ }^{6}$. The patterning of metal foils for patterned spring layers has been performed printed circuit board lithography and by laser cutting. Both of these techniques are capable of very large areas.

\subsection{Areal Density}

One of the goals of this project is to produce a mirror technology that can be made lightweight with an areal density on the order of $1 \mathrm{~kg} / \mathrm{m}^{2}$. Since the nanolamiante layers are thin enough to be flexible, they are also lightweight. A representative device with $15 \mu \mathrm{m}$ thick mirror layer, $7 \mu \mathrm{m}$ thick spring layer and a $200 \mu \mathrm{m}$ thick base layer has a areal density of $2.32 \mathrm{~kg} / \mathrm{m}^{2}$.

\subsection{Bonding}

Much of the bonding work in this project was performed by MicroAssembly Technologies. The original plan for this project was to use the metal compression bonding. However, the success of the die-attach epoxy used in early devices and the fact that it requires no additional heating made a compelling case for moving to using epoxy exclusively. Early devices showed significant print-through due to leakage of the epoxy away from the bonding location. The leakage has been minimized by reducing the volume of epoxy and increasing the accuracy of the deposition. This has resulted in greatly reduced print-through. The utility of epoxy bonding has been extended to large-pixel-count devices by the use of a screen printer. The screen printer allows the production of thousands of individual dots of epoxy on the order of $100 \mu \mathrm{m}$ wide.

\subsubsection{Counteracting the effects of curling by edge clamping:}

One of the most vexing problems in the development of nanolaminate-based deformable mirrors has been the difficulty in producing flat foils. It is critical that the residual moment in the foils be small enough that it can be overcome by the weak electrostatic actuators. We hypothesize that the primary cause of curling in nanolaminate foils is the heating of the foil during deposition. The top layers of the foil are deposited at a higher temperature than the bottom layers. When the foil temperature equilibrates, the different temperature changes through the thickness of the foil results in different thermal stresses. When the foil is released, the differences in stress result in curling. Considerable work has gone into producing nanolaminate foils that lay flat ${ }^{7,8}$. However, to date, the solution to nanolaminate flatness, pre-heating the substrate, is not robust. The heating of the substrate must be calibrated experimentally with day-long deposition runs and the system quickly goes out of calibration. The calibration must be redone each time any of the deposition parameters are changed. We are currently attempting use edge clamping of the mirror to counteract the moment that causes curling in the foils. This will allow the fabrication of functional nanolaminate deformable mirrors without requiring strictly flat foils.

In the absence of any other loads, equal and opposite moments applied at either end of a beam will produce a constant moment in the beam. If there is already a constant moment in the beam due to internal stresses, the moment applied at the ends can counteract that moment by superposition and bring the total moment in the beam to zero. In two dimensions a constant isotropic bending moment such as the thermally induced stress believed to cause curling in nanolaminate foils, can be counteracted buy clamping all the edges of the plate. 

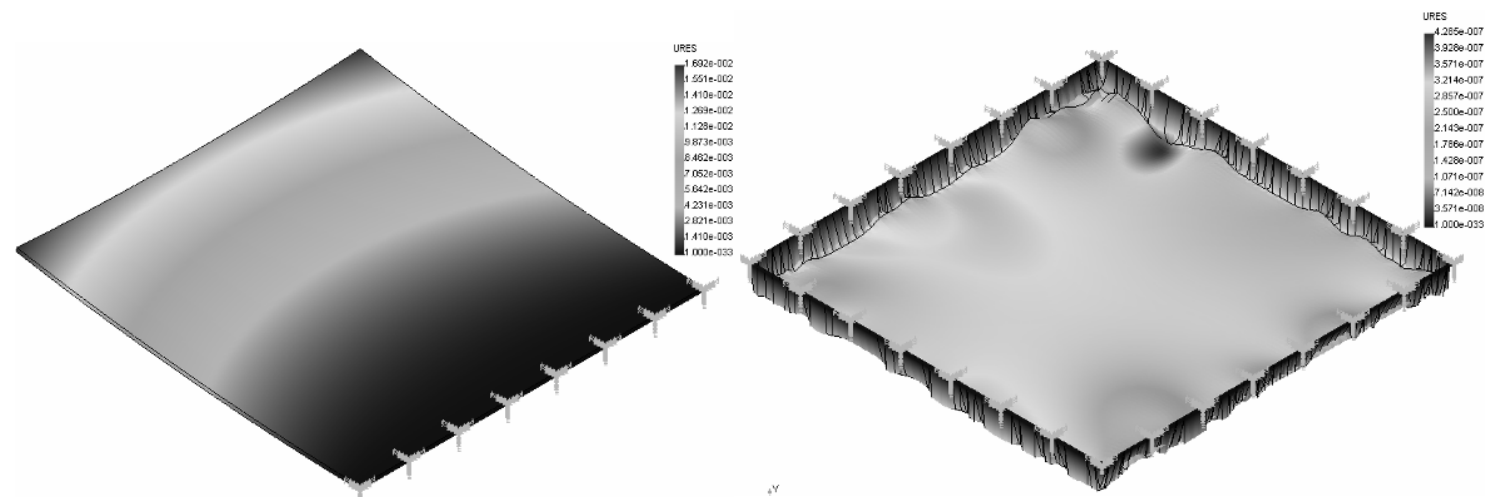

Figure 3. FEM analysis of a plate with an intrinsic moment. On the left, the plate is only clamped at one edge. On the right the plate is clamped on all four edges. The deformations of the fully clamped version are over 4 orders of magnitude smaller and result primarily from a peculiarity in the way the analysis was done. The plate was simulated as two flat plates with equal but opposite coefficients of thermal expansion. The upper plate, with a negative CTE shrunk and therefore moved downward relative to the perfectly stationary edges.

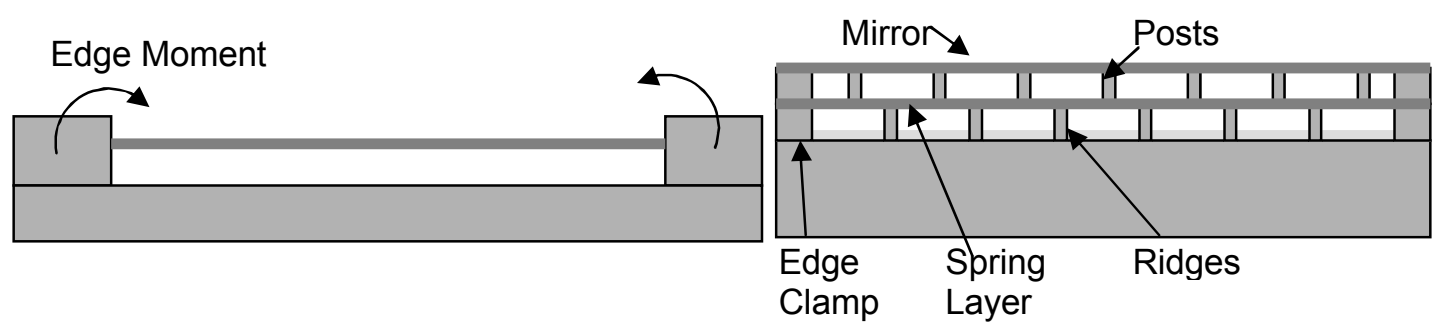

Figure 4. Cross section of 32x32 device with clamped edges.

\section{DESIGN}

\subsection{Model}

The modeling of this device has been described earlier ${ }^{7,8}$ but it will be summarized here. We produced a parametric model of the deformable mirror by first modeling the actuator, then the mirror, then combining the two. We then used the model to determine parameters for the design of the actuator that would yield the desired performance.

\subsubsection{Actuator model}

The first step in modeling the system is to model the actuator portion of the mirror. If all the actuators are actuated together, the mirror should move as a solid body and there should be no stress applied on the actuator by the mirror. Therefore, the displacement of an actuator by itself is a good estimation of the low-spatial frequency displacement.

\subsubsection{Patterned spring layer design}

The patterned spring layer was modeled as a parallel-plate actuator with linear springs. The springs were modeled as fixed-guided beams. The spring and electrostatic forces can be balanced with the force applied by the mirror to solve for displacement as a function of voltage.

\subsubsection{Continuous spring layer design}

The deformation of this actuator was modeled by balancing the strain energy in the deformed plate with the change in electrostatic energy in the capacitor between the deformed plate and the stationary electrode. The deformed plate was assumed to have the shape of a double cosine curve, the exact solution for small deformations with a uniform load. Although the deformation of the actual plate is likely to be different due to the concentration of electrostatic load near the center, this deviation will be small. 


\subsection{Mirror Model}

The deformation of a plate with arbitrary loads is the solution to a partial differential equation. Solving the PDE numerically is challenging. In addition, the model for the mirror is only valuable if it yields parametric information about the displacement of pixels relative to each other under loads. We simplified the system to a repeating high-spatialfrequency pattern for which a solution could be easily found and which provided a meaningful metric for relative displacement between pixels.

\subsubsection{Checkerboard pattern}

We chose to model the mirror with a "checker board" actuation pattern where every other actuator is pulled down with the maximum potential and the alternate actuators are not actuated. The checkerboard is a repeating high-spatialfrequency shape that can be easily modeled. It can be seen from inspection that each element in a checkerboard-loaded plate is a square plate with a point load in the center and simply supported at each edge. The displacement between the loaded and unloaded pixels gives information about the ability of the system to make high-spatial frequency shapes.

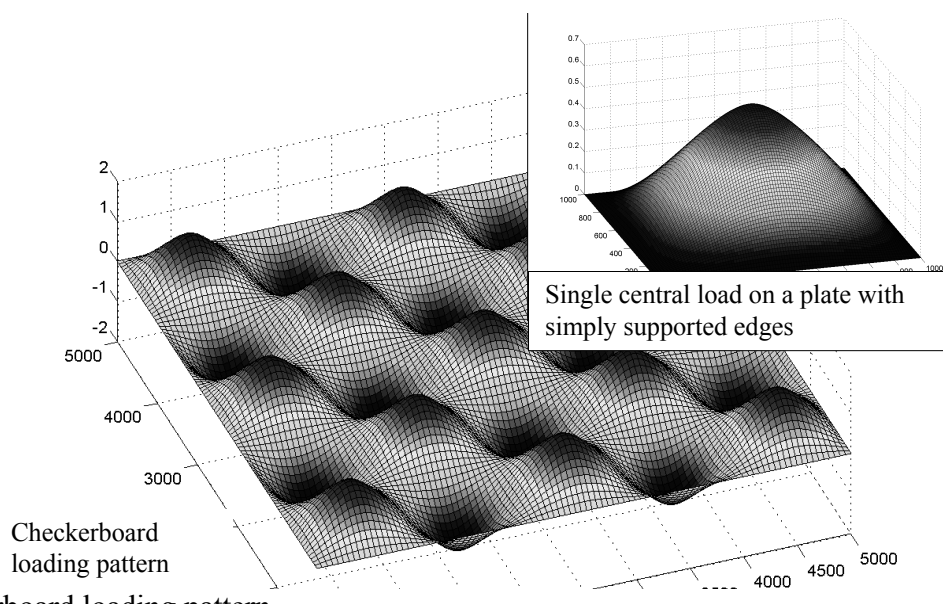

Figure5. Solution to checkerboard loading pattern

\subsection{Integrated system}

With models for the two major components of the deformable mirror, the next problem is to combine the two to find the high-spatial-frequency response of the entire system. One of the advantages of the checkerboard pattern is that there are an equal number of actuated and un-actuated pixels. Thus the force applied by each actuated pixel on the mirror must equal the force applied by the mirror on each un-actuated pixel.
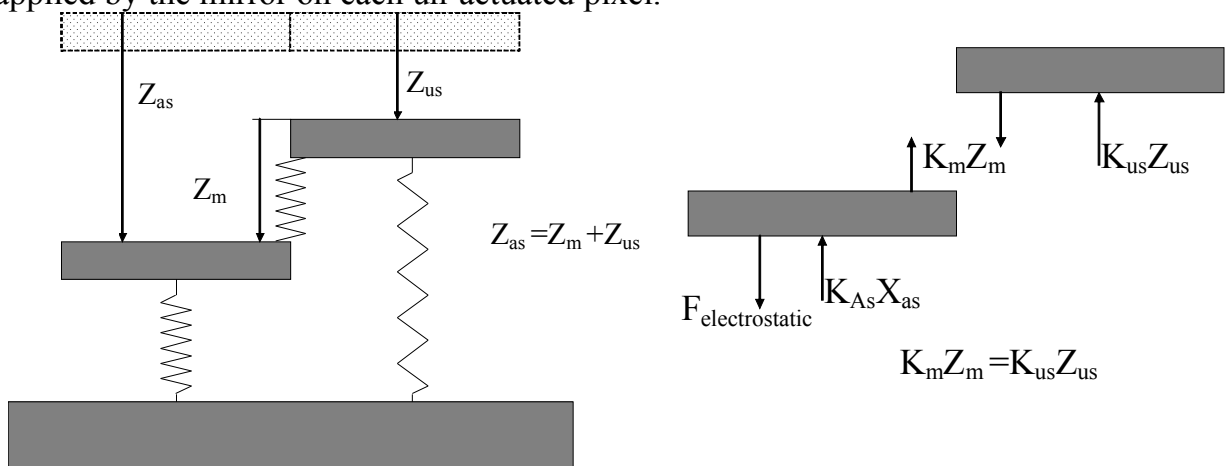

Figure 6.On the left is the schematic of the actuated/un-actuated pixel system. The dotted boxes indicate the rest position of both pixels, the grey boxes indicate the deformed position of the pixels. The pixel on the left is pulled down electrostaically, the pixel on the right is pulled down by the mirror. The mirror is represented by a sprig between the two pixles. On the right is the free-body diagram of the two pixels. 
As shown in the diagram above, the deflection of the actuated pixel, $Z_{\text {as }}$ must be equal to the sum of the deflection of the mirror $Z_{m}$ and the un-actuated pixel $Z_{u s}$. From the free-body diagram of the two pixels, the force translated through the mirror must be equal to the restoring force of the deformed mirror and the restoring force of the un-actuated pixel. From the actuator model, we can find the electrostatic force as a function of displacement. This gives us 3 equations and 3 unknowns, so we can solve for the displacement of all three portions of the system and can find the high-spatialfrequency displacement.

We estimated intermediate spatial frequencies by scaling the results for high-spatial-frequency. We multiplied the stiffness and actuation force of the actuators by the number of pixels ganged together. Since plate stiffness is proportional to inverse length squared we divided the mirror stiffness by the spacing between the centers of groups of actuators ganged together squared.

\subsection{Design}

We used the model to pick parameters that would yield both sufficient high-spatial-frequency displacement while providing sufficient low-spatial-frequency displacement and keeping the foils thick enough to be "handleable."

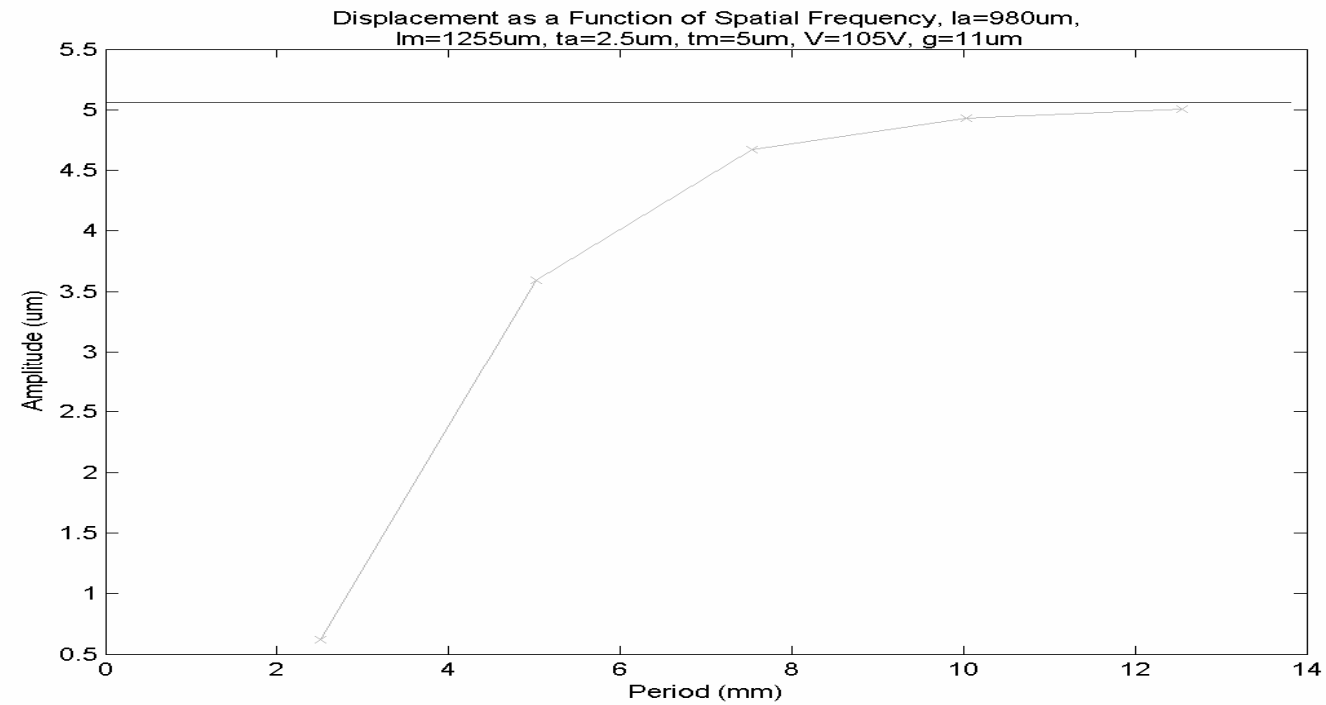

Figure 7. Predicted performance of $32 \times 32$ device. Low spatial frequency displacement is $5 \mu \mathrm{m}$, high-spatial-frequency displacement is just under $1 \mu \mathrm{m}$. Intermediate spatial frequencies produce intermediate displacements.

\section{FABRICATION}

This project has been executed in a phased approach starting with a small array made of convenient materials working up to a two final products: a 10 -across DM with pixel spacing on the order of $1 \mathrm{~cm}$ and a 1000 actuator DM with pixel spacing on the order of $1 \mathrm{~mm}$.

\begin{tabular}{|l|l|l|l|l|l|}
\hline & $1^{\text {st }} 3 \times 3$ & $2^{\text {nd }} 3 \times 3$ & $1-\mathrm{cm}$ & 10 -across & $32 \times 32$ \\
\hline Pixel Count & 9 & 9 & 4 & 76 & 1024 \\
\hline Pitch & $1 \mathrm{~cm}$ & $1 \mathrm{~cm}$ & $5 \mathrm{~mm}$ & $9 \mathrm{~mm}$ & $1.255 \mathrm{~mm}$ \\
\hline Base Material & Glass & Silicon & Silicon & Silicon & Silicon \\
\hline Ridge Material & SU-8 epoxy & $\begin{array}{l}\text { Electroplated } \\
\text { Nickel }\end{array}$ & $\begin{array}{l}\text { Electroplated } \\
\text { Nickel }\end{array}$ & $\begin{array}{l}\text { Electroplated } \\
\text { Nickel }\end{array}$ & $\begin{array}{l}\text { Electroplated } \\
\text { Nickel }\end{array}$ \\
\hline Spring Layer & Rolled Invar & Nanolaminate/ & $15 \mu \mathrm{m}$ & $15 \mu \mathrm{m}$ & $2.5 \mu \mathrm{m}$ \\
\hline
\end{tabular}




\begin{tabular}{|c|c|c|c|c|c|}
\hline & foil & Rolled invar & Nanolaminate foil & Nanolaminate & Nanolaminate foil \\
\hline Post material & SU-8 epoxy & $\begin{array}{l}\text { Electroplated } \\
\text { Nickel }\end{array}$ & $\begin{array}{l}\text { Electroplated } \\
\text { Nickel }\end{array}$ & $\begin{array}{l}\text { Electroplated } \\
\text { Nickel }\end{array}$ & $\begin{array}{l}\text { Electroplated } \\
\text { Nickel }\end{array}$ \\
\hline Mirror Material & $\begin{array}{l}\text { Rolled Invar } \\
\text { Foil }\end{array}$ & $\begin{array}{l}15 \mu \mathrm{m} \\
\text { Nanolaminate foil }\end{array}$ & $\begin{array}{l}15 \mu \mathrm{m} \\
\text { Nanolamiante foil }\end{array}$ & $\begin{array}{l}15 \mu \mathrm{m} \\
\text { Nanolaminate foil }\end{array}$ & $\begin{array}{l}5 \mu \mathrm{m} \text { nanolaminate } \\
\text { foil }\end{array}$ \\
\hline
\end{tabular}

\subsection{First Generation $3 \times 3$}

The first generation device was a $3 \times 3$ array proof of principle device using materials that were easily obtainable and easily put together. The base layers of these devices were glass wafers. Ridges were formed on the wafers by patterning SU-8 epoxy. Rolled invar layers were used as the mirror and spring layers. Two devices were produced. One has a patterned spring layer the other has a continuous spring layer. Both of these devices were bonded with die attach adhesive.
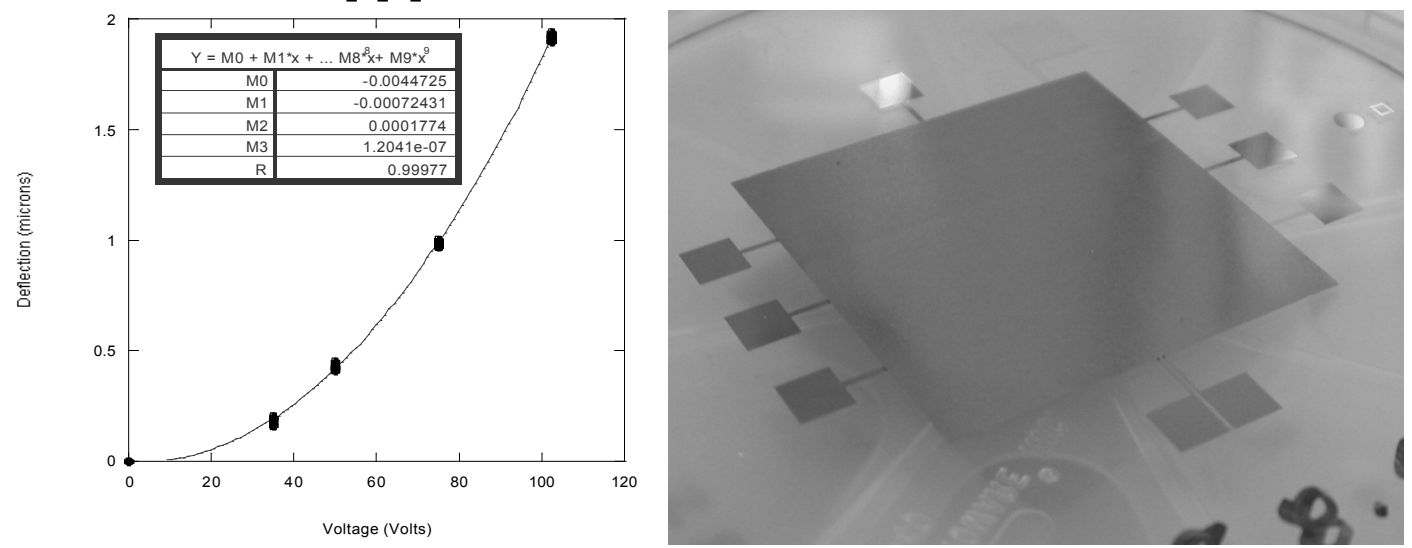

Figure 6. Deflection vs. Voltage for first generation device, photograph of first generation device.

\subsection{Second Generation $3 \times 3$}

The second generation $3 \times 3$ devices were fabricated with electroplated metal posts and ridges and with nanolaminate mirrors.

For these devices, nickel ridges were electroplated on a silicon wafer and nickel posts were fabricated on the back side of nanolaminate foils. Seven of these devices were produced with continuous nanolaminate foil spring layers. One device was produced with a patterned invar spring layer. One additional test device was produced with a nanolaminate spring layer but no mirror layer. The bonding techniques have been presented previously?

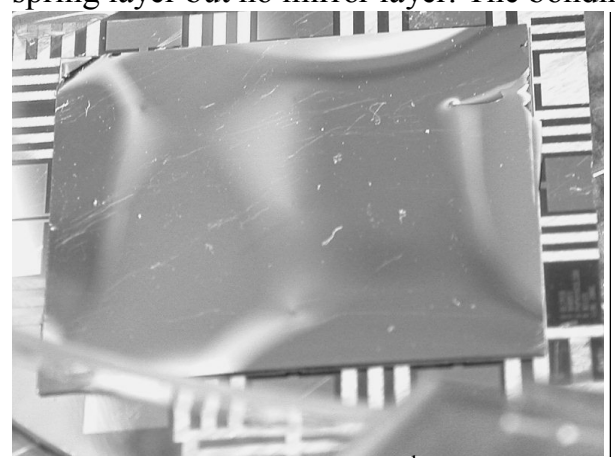

Displacement vs. Voltage Sample \#5 pixel \#1

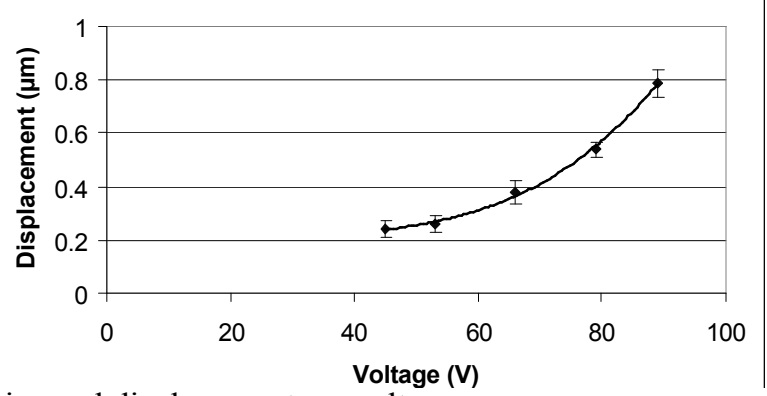

Figure 9. Photograph of a $2^{\text {nd }}$ generation device and displacement vs. voltage.

\section{3. $1-\mathrm{cm}$}


We produced very small 1-cm, 4 pixel mirrors as a way to develop the bonding process with a limited amount of nanolaminate foil. The development of a bonding process had been limited by the small number of suitable nanolaminate foils that could be produced. Each 4" wafer produced only 4 pieces that could be used for the $3 \times 3$ devices and only 1 piece for the 10-across device. In contrast each 4 " wafer produces 60 foils for these 1 -cm devices. While they were intended only as test material for the bonder, they also produced some functional devices.

\subsection{0-across}

Two of the 10-across 76 pixel devices have been fabricated with continuous nanolaminate spring and mirror layers. The first indications are that the pixels move; changing fringes have been observed in a white-light interferometer. A system is being developed to measure the high and low spatial frequency displacements. However that system is not complete and accurate measurements are not available.

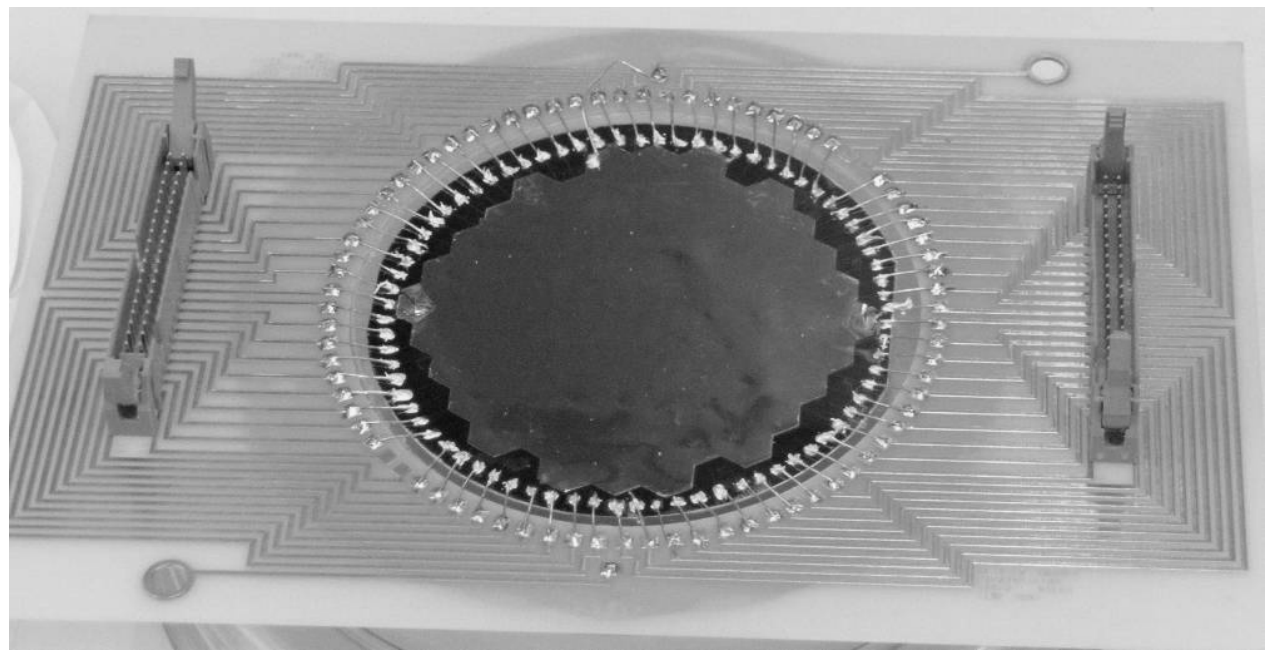

Figure 10. A completed 76-pixel, 10-across deformable mirror

\section{5. $32 \times 32$}

The last device is intended to show that the technology can be used for very dense arrays. This device is designed with 1024 pixels in a $32 \times 32$ array on a $1.255 \mathrm{~mm}$ pitch. The device is designed to use $2.5 \mu \mathrm{m}$ thick nanolaminate spring layers and $5 \mu \mathrm{m}$ thick nanolaminate mirror layers.

Scaling up to thousands of pixels will require a significant scale up in the number of epoxy dots. To apply these dots we have been developing a process to use a screen printer to make epoxy dots. Not only is this technology capable of creating an arbitrary number of dots in a few seconds, it is scalable to larger substrates.

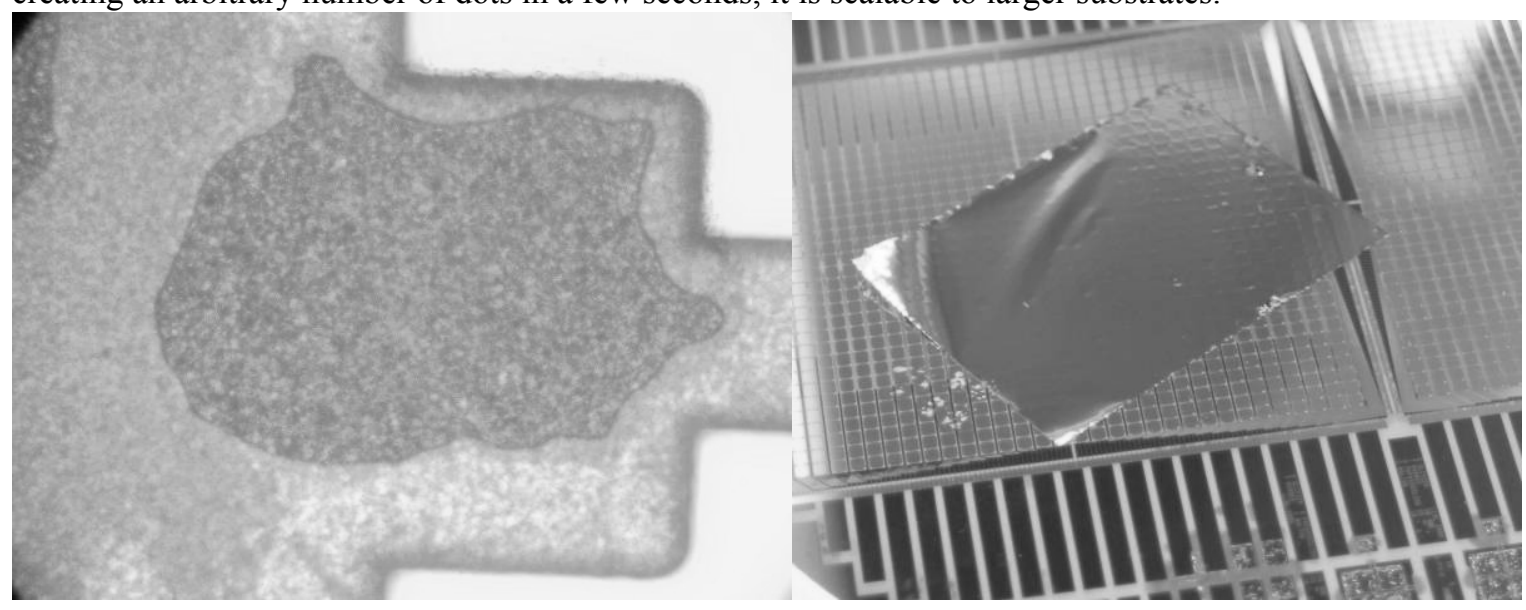


Figure 11. a) Epoxy bond between nickel ridges and a glass plate. b) A scrap piece of $2.5 \mu \mathrm{m}$ thick nanolaminate foil bonded to the ridges on a base layer.

\section{FUTURE WORK}

\subsection{Scaling to larger sizes}

There is no theoretical limit, only engineering limits, to the size that these devices could be made. The scaling of these devices up to the order of 1-meter has been discussed above. In order to scale to larger devices, one would need to develop systems to scale up 1) foil production, 2) lithography, 3) electroplating, 4) adhesive deposition, and 5) bonding. In many of these systems the cost of the machinery to produce a larger device will likely increase much more rapidly than the diameter of the mirror they are able to produce. Tiling many smaller pieces, or lithography or depositions may be the most cost-effective way to scale up to sizes larger than 1 meter.

\subsubsection{Nanolamiante foils}

The size of nanolaminate foils that can be fabricated is limited to the size of the vacuum chamber in which they are fabricated. The cost of fabricating the vacuum chamber and handling equipment likely would increase much more rapidly than the diameter of the largest usable foil. Another, perhaps more cost-effective option would be to tile together several moderately-sized foils.

\subsubsection{Lithography}

Current PC-board fabricating lithography systems are limited to the meter scale. However, there are at least two roads one could take to scaling up to much larger devices. It is possible to do lithography with laser systems. In this case, the size of the substrate is limited to the area an optical system can address, or the size of an X-Y table to move the substrate relative to a stationary laser. The other possibility is to tile lithography exposures to pattern a very large area. In this case the alignment of the individual tiles to each other and to the substrate becomes critical.

\subsubsection{Electroplating}

Electroplating can easily be scaled up to enormous sizes with brush electroplating. The fundamental limit is most likely a limit to the metrology. Since brush plating involves using the tool in different areas at different times, achieving the desired uniformity is challenging. The technique would require locally measuring the thickness of the plated metal and the ability to add or remove material to achieve uniformity.

\subsubsection{Adhesive deposition}

Adhesive deposition over a large area might be achieved by tiling screen-printed adhesive depositions. It might also be achieved with UV patterned adhesives.

\subsubsection{Bonding}

Much like the production of nanolaminate foils, the bonding of pieces to other piece is limited by the size of the machines used. Specialized bonding equipment would need to be developed at great expense. Again, tiling may be the cost effective solution

\subsection{Curved surfaces}

Most of the equipment available for the production of large nanolaminate foils is limited to work on flat surfaces. However, it may be possible to fabricate tools to work on curved surfaces. Nanolamiante foils can easily be fabricated in curved shapes merely by depositing the nanolaminates on a curved substrate. Lithography can be achieved on curved surfaces with laser systems. Electroplating can easily be achieved on curved surfaces. Adhesive deposition may be more challenging. Screen printing only works on flat surfaces. However, it may be possible to use a stamping system where adhesive is applied to a curved, patterned stamp and applied to a curved substrate. Bonding to a slightly curved substrate could also be fairly straight forward if the proper tools were developed.

\subsubsection{Reducing weight}


The vast majority of the mass associated with these devices is the mass of the base layer. Finding a way to make a base layer that is less massive would enable one to greatly reduce the mass of the system. The requirements of the substrate are that it is smooth with an RMS roughness on the order of a micron or less, sufficiently stiff to hold the shape of the mirror and that it have an insulating layer capable of resisting voltages on the order of $100 \mathrm{~V}$.

\subsubsection{Thinner substrates}

The substrates used in this research were all around $500 \mu \mathrm{m}$ thick, merely because that thickness was convenient. The thickness could be reduced until the base is only as thick as it absolutely needs to be.

\subsubsection{Stiffer or less dense material}

Silicon is a good choice of material for making a stiff, lightweight substrate. Silicon has a young's modulus near that of Titanium but has a lower density. However, switching to silicon carbide would allow a thinner, lighter substrate. Silicon carbide has a density similar to silicon and is around four times as stiff as Titanium.

\subsubsection{Supporting structures}

The base layers used to date have been flat sheets of material. However, substantial weight savings could be achieved by creating a ribbed structure or by stretching the base layer across a ring like a drum head.

\section{CONCUSION}

We have demonstrated a technology that can be scaled to the meter class and is capable of producing deformable mirrors with the required actuator density and deformations. We have fabricated small scale versions that demonstrate the potential of larger devices. We are currently fabricating a 10-across device with over $9 \mathrm{~cm}$ of clear aperture which will further demonstrate the practicality of this fabrication technique.

\section{ACKNOWLEDGEMENTS}

This work was performed under the auspices of the U.S. Department of Energy by University of California, Lawrence Livermore National Laboratory under Contract W-7405-Eng-48.

\section{REFERENCES}

1. Mansell, J., and Byer, R.; "Silicon Micromachined Deformable Mirror." SPIE-Int. Soc. Opt. Eng. Proceedings of Spie - the International Society for Optical Engineering, vol.3353, 1998, pp.896-901. USA

2. Kruczynski, P., Bogart, B., Lai, W., Lifton, V., Mansfield, W., Tyson, J., Sadoulet, B., and Williams, D., Electrostatically actuated membrane mirrors for adaptive optics, SPIE-Int. Soc. Opt. Eng. Proceedings of Spie - the International Society for Optical Engineering, vol.4983, 2003, pp.305-313. USA

3. Bifano T, Bierden P, Perreault J. Micromachined deformable mirrors for dynamic wavefront control. SPIE-Int. Soc. Opt. Eng. Proceedings of Spie - the International Society for Optical Engineering, vol.5553, no.1, 12 Oct. 2004, pp.1-16. USA.

4. Hickey G., Lih, S., Barbee, T. Development of nanolaminate thin-shell mirrors. SPIE-Int. Soc. Opt. Eng. Proceedings of Spie - the International Society for Optical Engineering, vol.4849, 2002, pp.63-76. USA.

5. Timoshenko, S. and Woinowsky-Krieger, S.; Theory of Plates and Shells, McGraw-Hill, NewYork, 1959

6. Hickey, G., Redding, D., Lowman, A., Cohen, D., Ohara, C., Actuated Hybrid Mirror Telescope NASA Techbriefs NPO-40105 2005

7. Papavasiliou, A., Olivier, S., Barbee, T., Miles, R., Chang, K., Large Scale Nanolaminate Deformable Mirror. SPIE-Int. Soc. Opt. Eng. Proceedings of Spie - the International Society for Optical Engineering, vol 6113, 2006, pp. 200-209

8. Papavasiliou, A., Olivier, S., Barbee, T., Walton, C., Cohn, M., "MEMS Actuated Deformabe Mirror" SPIE-Int. Soc. Opt. Eng. Proceedings of Spie - the International Society for Optical Engineering, vol 6113, 2006, pp. 190199

9. Cohn, M., Chang, K., Papavasiliou, A., Olivier, S., Kim, G., Matus, G. Xiao, Z., Cheung, S., Saechao, K. Roehnelt, R., "High performance adaptive optics using microscale assembly", SPIE-Int. Soc. Opt. Eng. Proceedings of Spie the International Society for Optical Engineering, vol 6113, 2006, pp. 162-170 